\title{
Three endoplasmic reticulum-associated fatty acyl-coenzyme a reductases were involved in the production of primary alcohols in hexaploid wheat (Triticum aestivum L.)
}

Guaiqiang Chai ${ }^{1,2}$, Chunlian $\mathrm{Li}^{1,2}$, Feng $X \mathrm{u}^{2}$, Yang $\mathrm{Li}^{2}$, Xue Shi ${ }^{1,2}$, Yong Wang ${ }^{1,2^{*}}$ and Zhonghua Wang ${ }^{1,2^{*}}$

\begin{abstract}
Background: The cuticle covers the surface of the polysaccharide cell wall of leaf epidermal cells and forms an essential diffusion barrier between the plant and the environment. The cuticle is composed of cutin and wax. Cuticular wax plays an important role in the survival of plants by serving as the interface between plants and their biotic and abiotic environments, especially restricting nonstomatal water loss. Leaf cuticular waxes of hexaploid wheat at the seedling stage mainly consist of primary alcohols, aldehydes, fatty acids, alkane and esters. Primary alcohols account for more than $80 \%$ of the total wax load. Therefore, we cloned several genes encoding fatty acylcoenzyme A reductases from wheat and analyzed their function in yeast and plants. We propose the potential use of these genes in wheat genetic breeding.
\end{abstract}

Results: We reported the cloning and characterization of three TaFARs, namely TaFAR6, TaFAR7 and TaFAR8, encoding fatty acyl-coenzyme A reductases (FAR) in wheat leaf cuticle. Expression analysis revealed that TaFAR6, TaFAR7 and TaFAR8 were expressed at the higher levels in the seedling leaf blades, and were expressed moderately or weakly in stamen, glumes, peduncle, flag leaf blade, sheath, spike, and pistil. The heterologous expression of three TaFARs in yeast (Saccharomyces cerevisiae) led to the production of C24:0 and C26:0 primary alcohols. Transgenic expression of the three TaFARs in tomato (Solanum lycopersicum) and rice (Oryza sativa) led to increased accumulation of C24:0-C30:0 primary alcohols. Transient expression of GFP protein-tagged TaFARs revealed that the three TaFAR proteins were localized to the endoplasmic reticulum (ER), the site of wax biosynthesis. The three TaFAR genes were transcriptionally induced by drought, cold, heat, powdery mildew (Blumeria graminis) infection, abscisic acid (ABA) and methyl jasmonate (MeJa) treatments.

Conclusions: These results indicated that wheat TaFAR6, TaFAR7 and TaFAR8 are involved in biosynthesis of very-longchain primary alcohols in hexaploid wheat and in response to multiple environmental stresses.

Keywords: Hexaploid wheat, Cuticular wax, Primary alcohol, Endoplasmic reticulum, Fatty acyl-coenzyme A reductase

\footnotetext{
*Correspondence: wangyong2114@163.com; zhonghuawang@nwafu.edu.cn

'State Key Laboratory of Crop Stress Biology for Arid Areas, Northwest A\&F

University, Yangling, Shaanxi 712100, China

Full list of author information is available at the end of the article
} 


\section{Background}

Plants are affected by poor environmental conditions, which interrupt normal growth and productivity. Drought is one of the most important abiotic stresses which causes osmotic stress in plants and profoundly affects crop production worldwide each year [1]. Plants respond to abiotic stresses by changing the varieties of cellular processes [2-4], and wax secretion from the plant epidermal cells to cuticle represents one of these significant changes. The cuticle, which covers most of the surfaces of terrestrial plants, mainly consists of cutin and cuticular wax. Insoluble cutin is the primarily structural component of the cuticle and is a polymer consisting of $\omega$ - and midchain hydroxy and epoxy $C_{16}$ and $\mathrm{C}_{18}$ fatty acids connected by ester bonds and glycerol bridges. Insoluble cutin constitutes $40-80 \%$ of the cuticle mass [5-7]. Cuticular waxes are complex mixtures of lipids that are composed of very-long-chain aliphatic molecules and theirs derivative, including primary and secondary alcohols, aldehydes, alkanes, ketones, esters, triterpenoids, sterols and flavonoids $[8,9]$. The cuticular wax plays an important role in the survival of plants, serving as the interface between plants and their biotic and abiotic environments. The primary physiological function of cuticular wax is to seal the tissue against a relatively dry atmosphere, preventing desiccation by minimizing nonstomatal water loss $[10,11]$. In addition, cuticular wax protects plants from UV radiation [12-15]. Cuticular wax also protects against plant pathogens and insects [16].

The biosynthesis of cuticular wax begins with $C_{16}$ or $\mathrm{C}_{18}$ de novo fatty acid synthesis on the outer membrane of epidermal cells in the plastid. Then, using $\mathrm{C}_{16}$ and $\mathrm{C}_{18}$ acyl-CoA and malonyl-CoA as substrates, the fatty acid elongase (FAE) complex performs a reiterative cycle of four reactions catalyzed by a $\beta$-ketoacyl-CoA synthase (KCS), a $\beta$-ketoacyl-CoA reductase (KCR), a $\beta$ hydroxyacyl-CoA dehydratase (HCD), and an enoyl-CoA reductase (ECR) to synthesize saturated very-long-chain fatty acids (VLCFAs) [17]. These VLCFAs are then further modified into various wax molecules through two major pathways: the acyl-reduction and the decarbonylation pathways [18]. Alcohols and esters are products in the acyl-reduction pathway, whereas alkanes, aldehydes, secondary alcohols and ketones are components of the decarbonylation pathway.

Bread wheat (Triticum aestivum L.2n $=42$; AABBDD) is a major food crop worldwide. At seedling stage, leaf cuticular waxes of hexaploid wheat mainly consist of primary alcohols, aldehydes, fatty acids, alkane and esters. Primary alcohols accounted for more than $80 \%$ of the total wax load, and $C_{28}$ primary alcohol is a major component of primary alcohols [19-21]. Thus, the acylreduction pathway mainly participates in this process.
Fatty-acyl coenzyme A reductase (FAR) is a crucial enzyme involved in the biosynthesis of very long chain fatty alcohols [22]. Several FARs from plants have been characterized, such as jojoba, Arabidopsis thaliana and wheat. In jojoba, the FAR could catalyze VLCFA precursors to fatty alcohols [23]. Eight genes encoding putative FARs have been identified in Arabidopsis, and the biological functions of five of the corresponding proteins have been described. AtFAR2 produces primary alcohols that are incorporated into sporopollenin of the pollen exine layer [24, 25], while CER4 (AtFAR3) generates C24:0-C30:0 primary alcohols present in the cuticular wax of aerial tissues [22]. Furthermore, three FARs (AtFAR1, AtFAR4 and AtFAR5) catalyze the formation of the 18:0, 20:0 and 22:0 fatty alcohols found in suberin polyester and root waxes [26]. Several FARs have been identified in gramineous model crops. In rice, DPW and OsFAR6/1/4 participated in primary alcohol biosynthesis [27, 28]. Brachypodium distachyon BdFARs have also proven to possess FARs activity [29]. In hexaploid wheat, TaFAR1, TaFAR5, TaTAA1a, TaFAR2, TaFAR3 and TaFAR4 have been identified. These genes all encoded FARs and participated in biosynthesis of fatty alcohols. TaFAR1 and TaFAR5 could produce C26:0, C28:0 and C30:0 fatty alcohols when expressing in tomato leaves [30, 31]. TaTAA1a, an anther-special gene, encodes a FAR related to pollen fertility [32]. TaFAR2, TaFAR3 and TaFAR4 catalyze the biosynthesis of C18:0, C28:0 and C24:0 fatty alcohols in yeast, respectively [33]. However, only a few genes produce $\mathrm{C}_{28}$ primary alcohol; therefore, other FAR genes may be involved in biosynthesis of fatty alcohols in wheat.

To further elucidate the molecular mechanisms of the other FARs from wheat, the aims of our study were as follows: (1) to clone and characterization three TaFARs from hexaploid wheat; (2) to identify whether these FARs to encode a functional FAR enzyme that catalyzes the production of very-long-chain primary alcohols in yeast; (3) to identify whether these TaFARs are responsible for fatty alcohol formation in transgenic tomato (Solanum lycopersicum cv. MicroTom) and rice (Oryza sativa L.); (4) to determine their subcellular location and expression patterns.

\section{Methods}

\section{Plant materials and growth conditions}

Hexaploid wheat Xinong979, tomato variety Micro-Tom (Solanum lycopersicum L.) and rice variety Zhonghua11 (Oryza sativa L. spp. japonica) were kindly provided by Crop Molecular Biology and Breeding laboratory, College of Agronomy, Northwest A\&F University, China. Among these materials, Xinong 979, a major winter wheat cultivar planted in the southern area of the Huang-huai wheat region in China, was used throughout 
the experiments for gene cloning and expression. Tomato variety Micro-Tom and rice variety Zhonghual1 were used for genetic transformation. For gene expression analysis, Xinong979 was grown in field during the 2014-2015 wheat-growing seasons. The young leaves were collected at seedling stage, and the root, peduncle, flag leaves, sheath, glume, spike, anther and pistil were collected at flowering stage. For abiotic stress analysis, the Xinong979 seedlings were grown in a greenhouse for 4-5 weeks and then subjected to various stresses. All abiotic stress treatments were conducted as described previously [31].

\section{Cuticular wax extraction, chemical components and scanning electron microscopy (SEM) analysis}

The tomato leaves and fruits, rice leaves and sheaths were harvested and immersed in chloroform for $50 \mathrm{~s}$ in a fume cupboard. After extraction, $20 \mu \mathrm{g} n$-tetracosane was added to each sample as an internal standard. The samples were transferred to a gas chromatograph (GC) autosampler vial and dried under a stream of nitrogen gas. For GC analysis, the wax samples were derivatized with $45 \mu \mathrm{L}$ pyridine (Sigma) and $45 \mu \mathrm{L} \mathrm{N}$,O-bis (trimethylsilyl)-trifluoroacetamide (BSTFA) (Sigma) for $1 \mathrm{~h}$ at $70{ }^{\circ} \mathrm{C}$. Then, the sample was dried under nitrogen gas before being redissolved in $500 \mu \mathrm{L}$ of chloroform. The GC equipped with a mass spectrometric detector was used for qualitative analysis, and flame ionization detector (FID) was used for quantitative analysis.

For SEM analysis, the cuticular tissues were harvested and dried under $50{ }^{\circ} \mathrm{C}$ for 1 week before $5-10 \mathrm{~mm}$ completely dried pieces were attached with double adhesive tape to the aluminum stubs and sputter-coated with gold particles. The samples were observed using a SEM (Hitachi S4800) at an accelerating voltage of $10 \mathrm{kV}$ and a working distance of $12 \mathrm{~mm}$.

\section{RNA isolation, cDNA synthesis and quantitative real-time PCR (qRT-PCR) analysis}

Total RNA from hexaploid wheat samples was extracted using TRIzol ${ }^{\mathrm{m}}{ }^{\text {Reagent }}$ (TaKaRa Biotechnology, Dalian Co., Ltd., China) according to the manufacturer's instructions. The potential contaminating DNA in total RNA was digested with DNase I. First-strand cDNA was synthesized using the GoScript Reverse Transcription System (TaKaRa). Oligo(dT)18 was used as a primer. The reverse transcription reaction was incubated at $42{ }^{\circ} \mathrm{C}$ for $1 \mathrm{~h}$ followed by $70{ }^{\circ} \mathrm{C}$ for $15 \mathrm{~min}$ in a total volume of $20 \mu \mathrm{L}$. After a 1:20 dilution, $0.5 \mu \mathrm{L}$ of the synthesized cDNA was used for RT-PCR or qRT-PCR. The primers for PCR were designed based on the cDNA sequence (Additional file 1: Table S1), and qRT-PCR was conducted as described previously [34]. To standardize the data, the wheat ACTIN gene (TaACTIN GenBank accession number AB181991) was used as an internal reference for the qRT-PCR analysis. Quantification of gene expression was performed using a CFX96 Real-Time PCR System (Bio-Rad). Dissociation curves were generated for each reaction to ensure specific amplification. All reactions, including the negative control, were performed three times. The threshold values $(C T)$ generated from the CFX96 Real-Time software tool were employed to quantify the relative gene expression using the comparative threshold $\left(2^{-\Delta \Delta C T}\right)$ method [35]. Three independent biological replicates were also performed for each experiment.

\section{Cloning and comparative analyses of TaFAR6, TaFAR7 and TaFAR8}

The protein sequence of CER4 (NC_003075) reported by Rowland [22] was aligned in National Center of Biotechnology Information (NCBI) via tBlastn. The protein shared high homologous identity with the uncharacterized proteins derived from wheat, but the function of the proteins was unknown. Thus, three pairs of special primers (Additional file 1: Table S1) were designed for amplification of TaFARs. The PCR products were purified, cloned into the pMD18-T vector (TaKaRa) and sequenced.

The cDNA sequences were analyzed using Lasergene7 (DNASTAR) and blast (https://blast.ncbi.nlm.nih.gov/ Blast.cgi) to search the Non-Redundant (NR). Pfam (http://pfam.xfam.org/), InterProScan (http://www.ebi.ac. uk/interpro/search/sequence-search) and PROSITE Scan (https://prosite.expasy.org/scanprosite/) were used to predict conserved domains or motifs. Multiple sequence alignments were performed by ClustalW. Phylogenetic trees of FARs were constructed by MEGA7.0 software.

\section{Heterologous expression of TaFARs in yeast (Saccharomyces cerevisiae)}

The coding sequences of TaFARs were amplified from cDNA of wheat cv. Xinong979 using a pair of primers labeled as pYES-TaFARs-7F and pYES-TaFARs-7R (Additional file 1: Table S1). The PCR fragment was cloned into the yeast expression vector pYES3. Then, the recombinant plasmid was transformed into $E$. coli $\mathrm{DH} 5 \alpha$ and verified by sequencing. Subsequently, the two vectors pYES3-TaFARs and P416 were co-transformed into yeast mutant strain INVSc1 (MAT $\alpha$ his3- $\Delta 1$ leu2 trp1-289 ura3-52), and the empty pYES3 vector was used as the negative control. Transgenic yeast cells were selected by growth on uracil and tryptophan deficient synthetic complete (SC-Ura-Trp) medium with $2 \%(w / v)$ glucose at $30{ }^{\circ} \mathrm{C}$. Three individual colony were induced and lipids were extracted using the protocol previously reported [36].

\section{Overexpression of TaFARs in tomato and rice}

To generate the overexpression vector for TaFAR6, TaFAR7 and TaFAR8, the entire ORFs of three FAR 
genes were amplified using primer pair TaFARs-F/ TaFARs-R and cloned into pCXSN, which was driven by the Cauliflower Mosaic Virus (CaMV) 35S promoter. The overexpression vectors were introduced into Agrobacterium tumefaciens strain GV3101 and EHA105. The resulting strains were used to transform the tomato cv. MicroTom and japonica cv. Zhonghua 11 as previously reported [37, 38]. All transgenic plants were planted in greenhouse. The phenotypes and cuticular wax analysis of the transgenic plants were examined in the $T_{1}$ generation.

\section{Subcellular localization of TaFARs}

The coding sequences of TaFAR6, TaFAR7 and TaFAR8 without the termination codons were cloned and recombined into the $\mathrm{N}$-terminus of sequences encoding specific fluorescent proteins under the control of the CaMV35S promoter. Briefly, the endoplasmic reticulum (ER) marker mCherry-HDEL and the fusion construct TaFARs-GFP were used in co-localiztion assays. Vectors were transferred into epidermal cell of tobacco using the bombardment-mediated method $[39,40]$ and incubated for $24 \mathrm{~h}$ in the dark at room temperature to allow transient expression. The transformed epidermal cells were observed using a confocal laser scanning microscope (LSM700; Zeiss, Germany) at the following excitation wavelengths: mCherry at $564 \mathrm{~nm}$ and GFP at $488 \mathrm{~nm}$.

\section{Results}

\section{Identification of $F A R$ genes from hexaploid wheat and} phylogenetic analysis

The previous studies indicated that the primary alcohols were the dominant components of cuticular wax on wheat leaves at the seedling stage [31, 41]. In order to clone the genes related to fatty alcohol biosynthesis, a Blast search of the wheat GenBank database was performed using the amino acid sequence of arabidopsis CER4 (GenBank accession No. NP_567936) which encodes an alcohol-forming FAR. Finally, ten candidate sequences were identified, and all exhibit high similarity to CER4 over their entire length. Six of these candidate sequences, TaFAR1, TaTAA1a, TaTAA1b, TaTAA1c, TaFAR2 and TaFAR3, have been reported, but the biological function of the other four genes have not been identified. The most highly represented genes, TaFAR6, TaFAR7 and TaFAR8 (GenBank accession No.MF8 04951, MF817443, MF817444), were selected for further research in this study. Subsequently, the cDNA fragments encoding TaFAR6, TaFAR7 and TaFAR8 were isolated from leaf blades of hexaploid wheat cv. Xinong979. Sequence analysis revealed that TaFAR6, TaFAR7 and TaFAR8 contain open reading frames of 1497, 1503 and 1479 bp that encode encoding 499, 501 and 493 amino- acid residues with the predicted protein molecular weight of 56.9, 57.4 and $55.6 \mathrm{kD}$, respectively.

The functional domain for TaFARs was predicted using online CDD software from the NCBI (https:// www.ncbi.nlm.nih.gov/Structure/cdd/wrpsb.cgi). The results showed that three TaFARs proteins contain a NADB binding domain at the $\mathrm{N}$-terminal linked with a FAR_C domain at the C-terminal. These two domains were consistent with FARs from other animal and plant species, such as Euglena EgFAR [42] and wheat TaFAR1 and TaFAR5 [30, 31]. Multiple sequence alignment revealed that TaFAR6, TaFAR7 and TaFAR8 exhibited $50-55 \%$ identity with five different plant species homologues: AtCER4, TAA1b, ZmFAR3, BdFAR3 and OsFAR1 (Fig. 1b). Phylogenetic analysis of 55 proteins with similarities to TaFAR6, TaFAR7 and TaFAR8 showed that all proteins can be grouped into three clades. TaFAR6, TaFAR7 and TaFAR8 were grouped into the first clade, which was derived from the monocot (Fig. 1a). TaFAR8 had the highest homology with three rice proteins OsFAR6, OsFAR1 and OsFAR4. TaFAR6 and TaFAR7 were the most similar to HvFAR2 and BdFAR3, respectively. These results suggest that the TaFAR6, TaFAR7 and TaFAR8 proteins possess FAR activity associated with primary alcohol biosynthesis in wheat.

\section{Analysis of TaFARs gene expression}

RT-PCR and qRT-PCR were performed to investigate the transcription profile of the three TaFARs in various whole tissues. The samples were derived from 2-monthold seedling leaves and various organs at the flowering stage. The TaFARs were expressed in all tissues except root tissue. TaFAR6 was highly expressed in seedling leaf blades and glumes, modestly expressed in peduncle and flag leaf blade, and weakly expressed in sheath, spike, stamen and pistil. TaFAR7 mainly showed a relatively high expression level in seedling leaf blades, spike and stamen compared with other tissue organs. TaFAR8 exhibited increased expression in the seedling leaf blade and was highly expressed in flag leaf blade, sheath and stamen (Fig. 2a, b). Furthermore, we generated a bacterial expression vector by inserting the TaFAR coding region into the pET28a vector (Novagen) and introducing this construct into E. coli BL21 (DE3). Consistent with the predicted size, the molecular mass of TaFAR6, TaFAR7 and TaFAR8 proteins were estimated to be 56.8 , 57.4 and $55.6 \mathrm{kD}$, respectively, by SDS-PAGE analysis (Additional file 2: Figure S2).

Previous studies indicated that the plant cuticular wax can be regulated by a range of abiotic stresses [43-45]. Transcript profiling from leaves of pot-grown wheat plants showed that TaFAR6, TaFAR7 and TaFAR8 genes were responsive to dehydration, cold, heat, ABA hormone, powdery mildew infection and MeJa treatment 


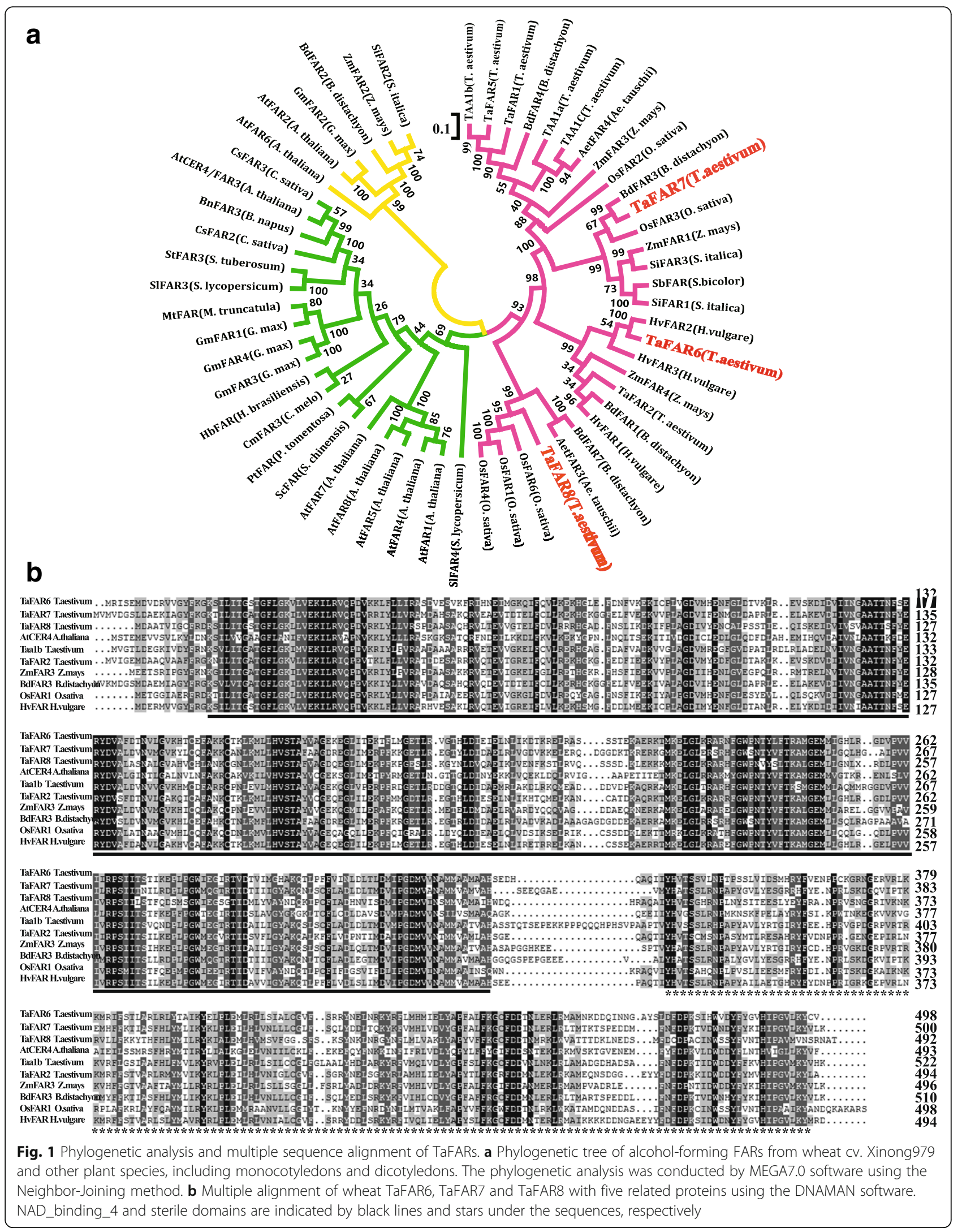



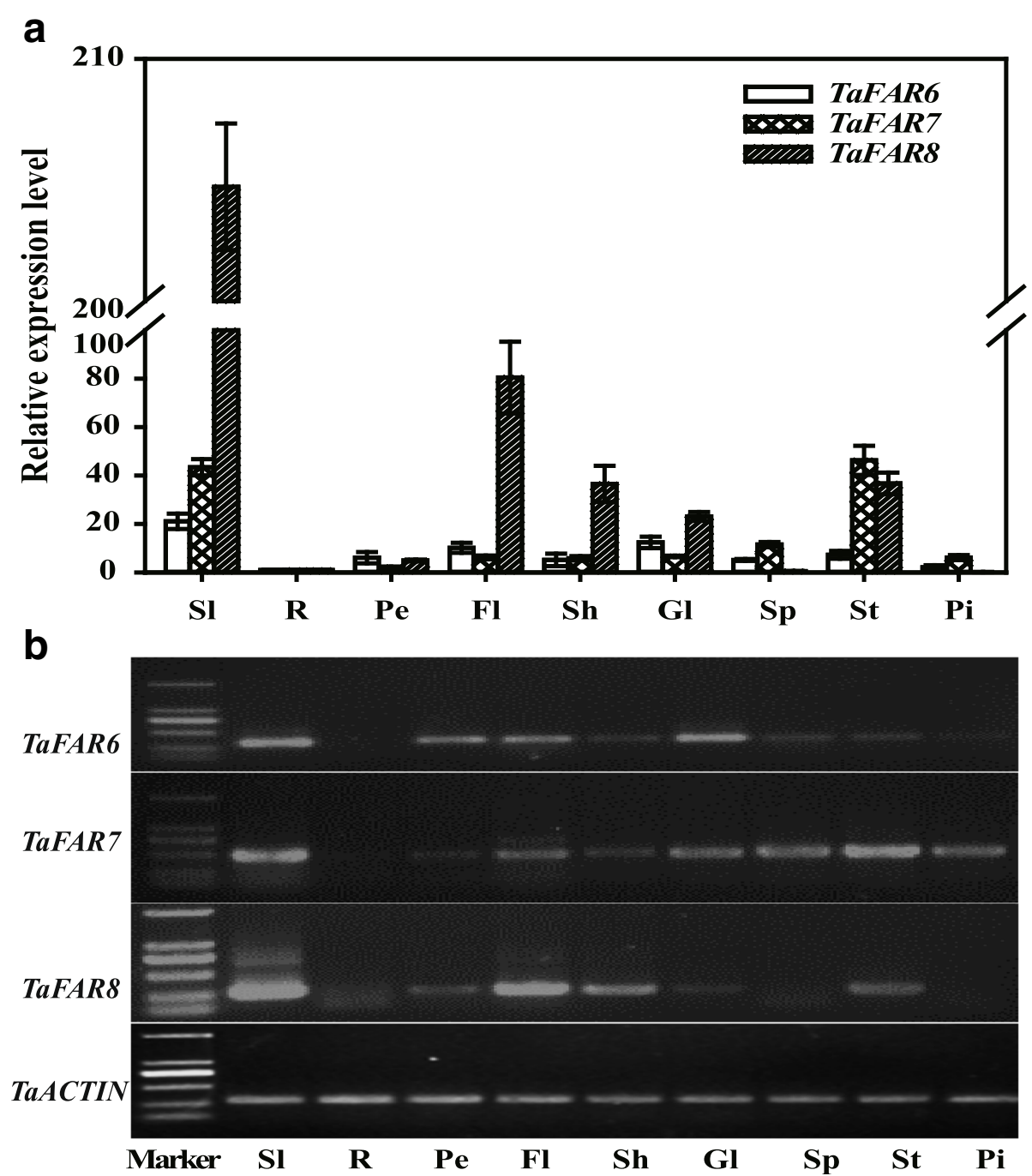

Fig. 2 Expression of TaFARs in different tissues of hexaploid wheat Cv. Xinong979. a qRT-PCR analysis of TaFARs expression in various organs of wheat. Sl, seedling leaf blade; R, root; Pe, peduncle; Fl, flag leaf blade; Sh, sheath; Gl, glume; Sp, spike; St, stamen; Pi, pistil. Wheat TaACTIN was used as reference. Error bars indicate the SE of three independent replicated samples. $\mathbf{b}$ Semi-quantitative analysis of TaFARs expression in various organs of wheat

(Fig. 3). When the seedlings were exposed to dehydration stress, TaFAR7 transcript levels peaked at $2 \mathrm{~h}$, whereas those of TaFAR6 and TaFAR8 peaked at $1 \mathrm{~h}$ (Fig. 3a). After ABA treatment, TaFAR7 and TaFAR8 showed the highest expression levels at $4 \mathrm{~h}$, while TaFAR6 peaked at $6 \mathrm{~h}$ (Fig. 3b). Expression of TaFAR7 slowly increased within $12 \mathrm{~h}$ after initiation of cold treatment, and its transcript level peaked at $24 \mathrm{~h}$. However, the accumulation of TaFAR6 and TaFAR 8 mRNA rapidly increased, and their expression levels peaked at $1 \mathrm{~h}$ and 6 h, respectively (Fig. 3c). Under heat stress condition, three TaFARs were induced, and TaFAR6 mRNA reached its highest level at $1 \mathrm{~h}$. In contrast, the highest transcript levels for TaFAR7 and TaFAR8 occurred at $12 \mathrm{~h}$ and $6 \mathrm{~h}$, respectively (Fig. 3d). TaFAR6, TaFAR7 and TaFAR 8 expression increased gradually within $24 \mathrm{~h}$ after powdery mildew infection and then rapidly declined over $24 \mathrm{~h}$ (Fig. 3e). Finally, we also exposed wheat seedlings to MeJA, which acts as an essential signal involved in defense/stress pathway in monocots [46, 47]. TaFAR6, TaFAR7 and TaFAR8 expression was strongly induced by MeJA and peaked at $1 \mathrm{~h}$. Compared with TaFAR6 and TaFAR8, TaFAR7 transcript levels dramatically increased until $6 \mathrm{~h}$ after MeJA treatment and peaked at $12 \mathrm{~h}$ (Fig. 3f). The results suggested that TaFAR6, TaFAR7 and TaFAR8 were induced by multiple environmental stresses and participated in the ABA and MeJA-dependent stress signal pathway.

\section{TaFAR expression in yeast}

In order to confirm the three TaFARs were alcoholforming FARs involved in the production of very-long- 

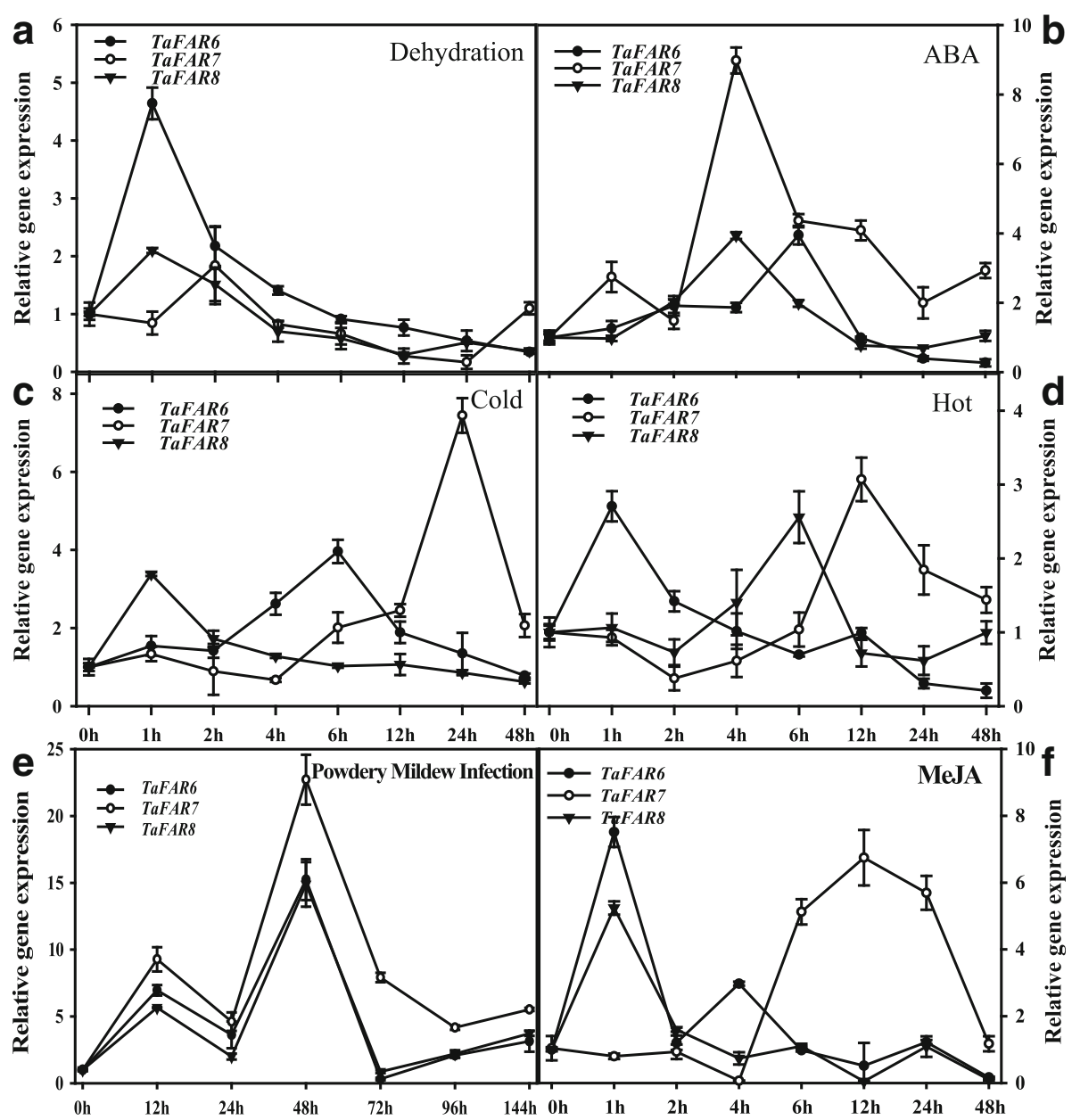

Fig. 3 Expression analysis of TaFAR6, TaFAR7 and TaFAR8 under various stresses conditions. Wheat cv. Xinong979 seedlings approximately four-week-old were exposed to stress treatments. a Dehydration treatments. b ABA treatment, $100 \mu \mathrm{M}$ ABA. c Cold treatment, $4{ }^{\circ} \mathrm{C}$. $\mathbf{d}$ Heat treatment, $42^{\circ} \mathrm{C}$ incubator. e Powdery mildew infection. $\mathbf{f}$ MeJA treatment, MeJA $100 \mu \mathrm{M}$

chain primary alcohols, we expressed the ORFs of three TaFAR genes in the yeast under the control of GAL1 promoter. The recombinant plasmid pYES3-TaFARs and p416 were co-transformed into the yeast mutant strain INVSc1 (Invitrogen), and the corresponding empty vector was used as the negative control. Yeast cells were induced and lipids were extracted according to the previous reports [48]. GC-MS results showed that no alcohols were detected when the empty vector was expressed in yeast (Fig. 4a). Based on authentic standards and MS characteristics, $\mathrm{C}_{24}$ and $\mathrm{C}_{26}$ primary alcohols were identified when TaFAR6 was expressed in transgenic yeast (Fig. 4b). Interestingly, $C_{24}$ and $C_{26}$ primary alcohols were also identified when TaFAR7 and TaFAR8 were expressed in transgenic yeast, respectively (Fig. 4c, d; Additional file 3: Figure S3). These results demonstrated that these three TaFARs possessed FAR activity and catalyzed the biosynthesis of very-long-chain primary alcohols.
The expression of three TaFARs in tomato results in primary alcohol accumulation

In order to further confirm that the TaFAR6, TaFAR7 and TaFAR8 were FARs catalyzing the accumulation of very-long-chain primary alcohols, we constructed three overexpression vectors pCXSN-TaFARs controlled by the cauliflower mosaic virus (CaMV) $35 \mathrm{~S}$ promoter (Additional file 4: Figure S4a), and these gene products were expressed in dicotyledon tomato and monocotyledon rice (Additional file 4: Figure S4b, e). The transgenic lines harboring empty vector were used as the negative control. No significant morphological differences were observed between transgenic lines and control lines (Additional file 4: Figure S4a). The cuticular wax composition of the red fruit and mature leaves from $\mathrm{T}_{1}$ generation transgenic lines were analyzed by GC-FID. As expected, the total primary alcohol content was dramatically increased in six transgenic lines compared with control lines, whereas the levels of fatty acid, aldehydes, 


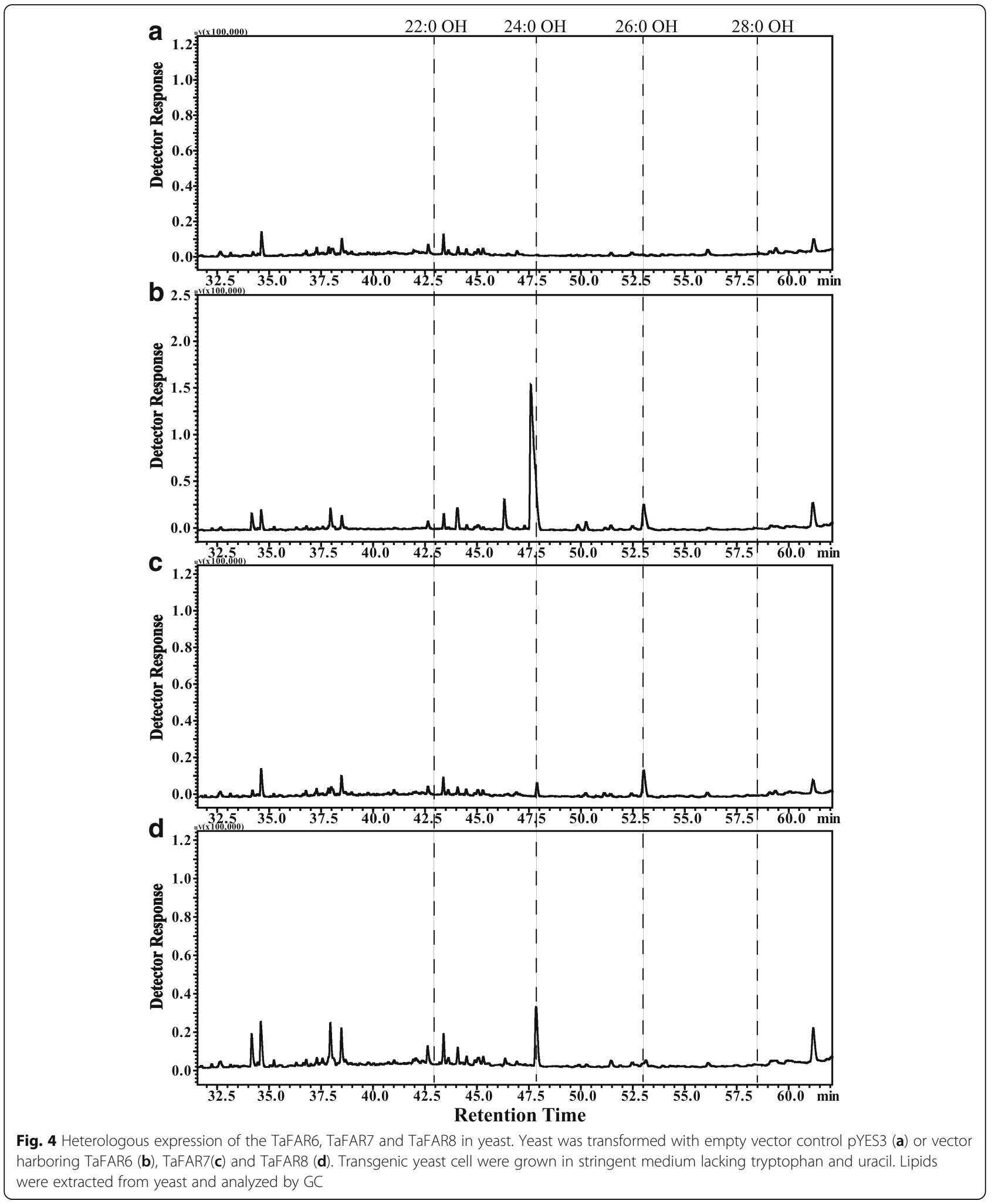

$n$-alkanes, branched alkanes, and triterpenoids were only slightly affected in the transgenic lines (Fig. 5a, c).

In fruits, the absolute content of total primary alcohols increased from $20.62 \mu \mathrm{g} / \mathrm{dm}^{2}$ in the fruit cuticle of the control line to $52.15 \mu \mathrm{g} / \mathrm{dm}^{2}$ in transgenic line TaFAR62, $77.20 \mu \mathrm{g} / \mathrm{dm}^{2}$ in line TaFAR7 -1 and $84.10 \mu \mathrm{g} / \mathrm{dm}^{2}$ in line TaFAR8-1 (Fig. 5a). Subsequently, the chain length of primary alcohols was further analyzed, and the results 

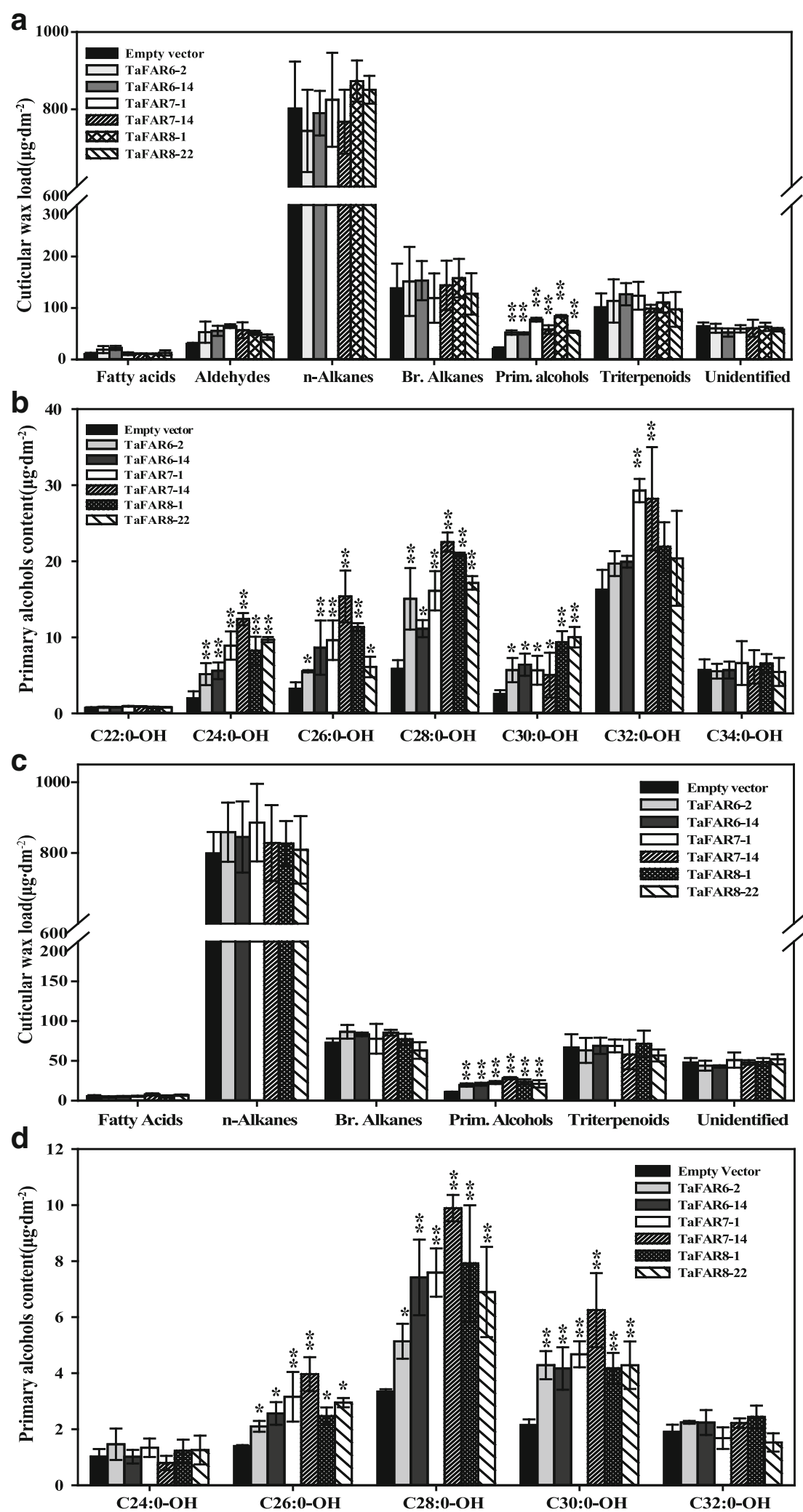

Fig. 5 (See legend on next page.) 
(See figure on previous page.)

Fig. 5 Cuticular wax analysis of tomato fruits and leaves overexpressing TaFAR6, TaFAR7 and TaFAR8. a Total fruit wax coverage in different compound classes. Data are expressed as $\mu \mathrm{g}$ per $\mathrm{dm}^{2}$. b Absolute amount $\left(\mu \mathrm{g} \cdot \mathrm{dm}^{-2}\right.$ ) of primary alcohols in fruits. c Total leaf wax coverage in different compound classes. $\mathbf{d}$ Absolute amount $\left(\mu \mathrm{g} \cdot \mathrm{dm}^{-2}\right)$ of primary alcohols in leaves. Values are means of $n \geq 4$ biological replicates. Error bars $=S D$. Asterisks represent significant differences between TaFAR expression vector and empty vector control (t-test: ${ }^{*}$ for $p<0.05$; ${ }^{* *}$ for $p<0.01$ )

revealed that $\mathrm{C}_{24}, \mathrm{C}_{26}, \mathrm{C}_{28}$ and $\mathrm{C}_{30}$ primary alcohols were increased in transgenic lines expressing TaFAR6 and TaFAR8 (Fig. 5b). Similar chain length patterns for primary alcohols were also observed in TaFAR7 transgenic lines except that the content of $\mathrm{C}_{32}$ primary alcohol was also dramatically increased compared with the control line. In the transgenic line TaFAR6-2, the greatest increase in the absolute contents of $\mathrm{C}_{24}, \mathrm{C}_{26}$, $\mathrm{C}_{28}$ and $\mathrm{C}_{30}$ primary alcohols, which exhibited increases of 1.6-, 0.7-, 1.5- and 1.2-fold, respectively, compared with the control line. Similar results were noted in the transgenic line TaFAR7-1. The wax mixtures on ripe fruit showed significant increases in primary alcohols with a 3.5-fold increase in $\mathrm{C}_{24}$ primary alcohol, a 2.0-fold increase in $\mathrm{C}_{26}$ primary alcohol, a 1.7 -fold increase in $\mathrm{C}_{28}$ primary alcohol, a 1.2 -fold increase in $\mathrm{C}_{30}$ primary alcohol, and a 0.8 -fold increase in $\mathrm{C}_{32}$ primary alcohol. Interestingly, the $\mathrm{C}_{24}, \mathrm{C}_{26}, \mathrm{C}_{28}$ and $\mathrm{C}_{30}$ primary alcohols were also found to be increased by 3.5-, 2.5-, 2.4- and 2.7-fold in the transgenic line TaFAR8-1, respectively (Fig. 5b).

In leaves, the wax load per unit of leave area of TaFAR6-2, TaFAR7-1 and TaFAR8-1 were 19.63, 22.75 and $23.51 \mu \mathrm{g} / \mathrm{dm}^{2}$, which is equivalent to 2-, 1.8-, 3-fold increase compared with the control lines, respectively (Fig. 5c). The chain length of primary alcohols was also further analyzed, and the results revealed that $C_{26}, C_{28}$ and $\mathrm{C}_{30}$ primary alcohols were dramatically increased in all transgenic lines (Fig. 5d).

In addition, in order to further determine whether the differences in the primary alcohol content in the transgenic lines influence the alteration of the crystal pattern, SEM revealed that substantially more cuticular wax crystals were deposited on the surface of the fruits epidermal cells in overexpression plants compared with CK plants (Additional file 5: Figure S5a-d). However, no evident crystal structure changes were observed on the surface of the epidermis of leaves (Additional file 5: Figure S5e-1). These primary alcohol changes provided further evidence that the three TaFARs are alcohol-forming FARs that are responsible for the very-long-chain alcohols formation in the epidermal cells of tomato fruits.

\section{Overexpression of three TaFARs altered the content of primary alcohols in rice}

To further confirm that the TaFARs were involved in cuticular wax biosynthesis, we also expressed these three TaFAR genes in rice cv. Zhonghua11. No significant morphological differences were noted between transgenic lines and CK lines (Additional file 4: Figure S4c), and cuticular wax composition of flag leaves from $\mathrm{T}_{1}$ generation transgenic lines were analyzed by GC-FID. As anticipated, a significant difference was detected on leaves of transgenic plants (Fig. 6a). Total primary alcohols were significantly increased, and other wax components exhibited no obvious changes. Then, the chain length of primary alcohols was further analyzed. The results revealed that $C_{24}$, $\mathrm{C}_{26}, \mathrm{C}_{28}$ and $\mathrm{C}_{30}$ primary alcohols were dramatically increased in transgenic lines of TaFAR6, TaFAR7 and TaFAR8, compared with the control line (Fig. 6b). In transgenic line TaFAR6-14, the greatest increases were noted in the absolute contents of $\mathrm{C} 24: 0-\mathrm{OH}, \mathrm{C} 26: 0-\mathrm{OH}$, $\mathrm{C} 28: 0-\mathrm{OH}$ and $\mathrm{C} 30: 0-\mathrm{OH}$, which exhibited increases of $4.2-, 1.8-, 0.4-$ and $0.3-$ fold, respectively, compared with control line. Interestingly, similar results occurred in transgenic line TaFAR7-12 and TaFAR8-2.

Subsequently,the sheath wax of rice was also detected. Similar results were found, and the wax mixtures on the sheath showed significant increases in primary alcohols but not in other compounds classes (Fig. 6c). The most drastic effect was observed for the $C_{28}, C_{30}$ and $C_{32}$ primary alcohols, all of which exhibited approximately 0.5 - to 1 -fold increase in independent transgenic lines compared with the control line (Fig. 6d). Finally, SEM was used for a detailed examination of the surfaces. In control lines, the adaxial and abaxial leaf blade surfaces were densely covered with platelet-type wax crystals including the unevenly distributed cuticular papillae. Transgenic rice lines overexpressing TaFAR6, TaFAR7 and TaFAR8 exhibited an obvious increase of wax crystals on both adaxial and abaxial sides of the leaf blade surface (Additional file 6: Figure S6a-h). Fortunately, there were also evident crystal structure changes on the surface of sheath epidermis (Additional file 6: Figure S6i-l). These results provide further confirmation that these three TaFARs were active FAR, likely accepting $C_{24}$ to $C_{30}$ fatty acyl-CoAs as substrates in rice.

\section{TaFARs located in the ER}

Because of the proteins that participate in the biosynthesis of cuticular wax are mainly located in the ER in plant epidermal cells $[17,49]$. We predicted that the three TaFARs should be localized to the ER. To confirm this prediction, we generated three constructs harboring the full length opening reading frame without termination codons of TaFARs fused upstream of the green 

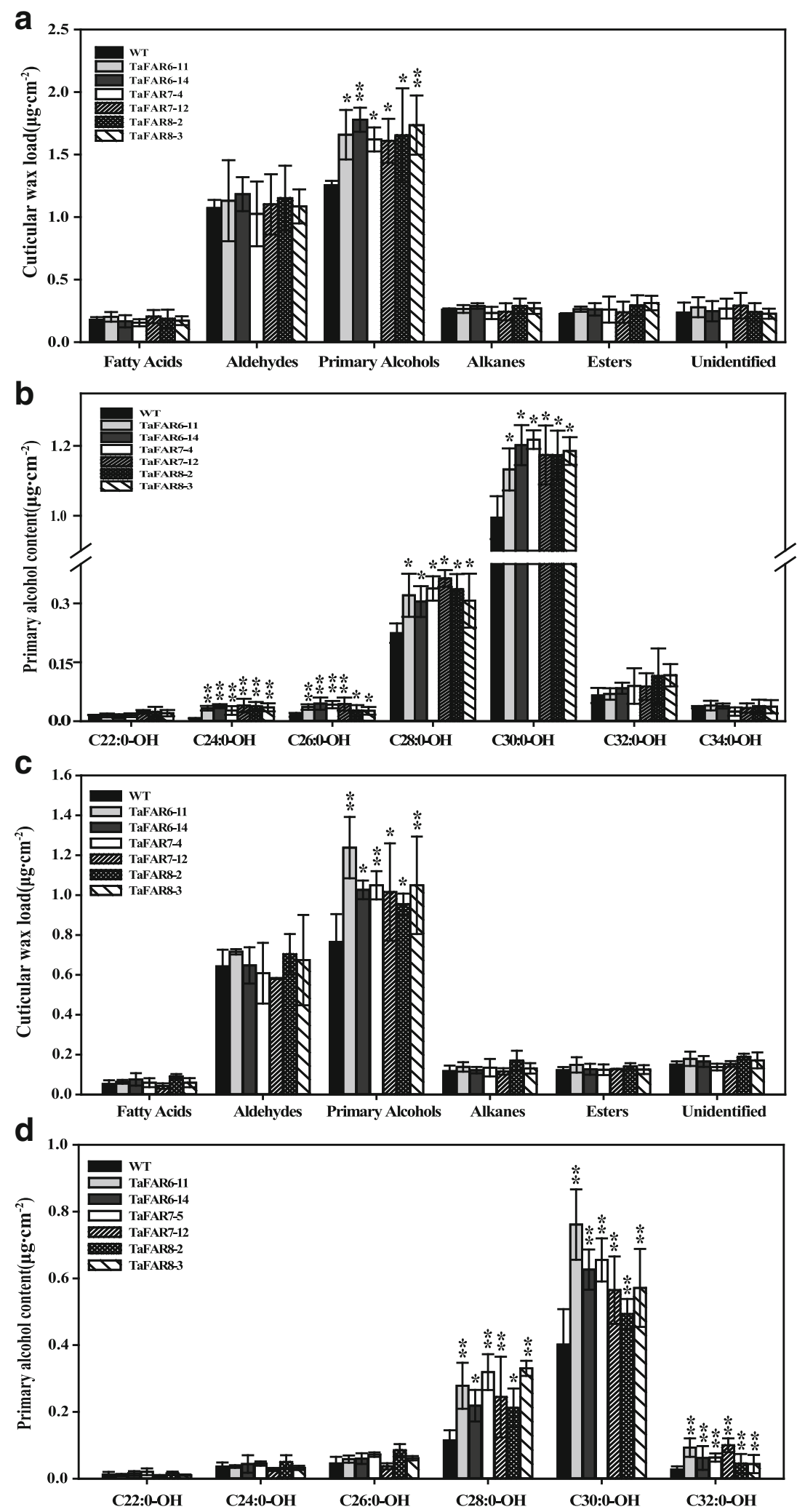

Fig. 6 (See legend on next page.) 
(See figure on previous page.)

Fig. 6 Cuticular wax analysis of rice leaf and sheath overexpressing TaFAR6, TaFAR7 and TaFAR8. a Total leaf wax coverage in different compound classes. Data are expressed as $\mu \mathrm{g}$ per $\mathrm{cm}^{2}$. b Absolute amount $\left(\mu \mathrm{g} \cdot \mathrm{cm}^{-2}\right)$ of primary alcohols in leaves. $\mathbf{c}$ Total sheath wax coverage in different compound classes. $\mathbf{d}$ Absolute amount $\left(\mu \mathrm{g} \cdot \mathrm{cm}^{-2}\right)$ of primary alcohols in sheath. Values are means of $n \geq 4$ biological replicates. Error bars $=S D$. Asterisks represent significant differences between TaFAR expression vector and empty vector control (Student's test was performed: * for $P<0.05$; ** for $P<0.01)$

fluorescent protein (GFP) gene under the control of CaMV $35 \mathrm{~S}$ promoter. In addition, a red fluorescent protein (RFP) mCherry-HDEL was used as an ER indicator $[50,51]$. These vectors were co-transformed into Nicotiana benthamiana leaf epidermal cells. Confocal microscopic observation showed that the green fluorescent signals of TaFARs were co-localized with the red fluorescent signals of ER-RFP (Fig. 7), suggesting that three TaFARs are ER-localized proteins.

\section{Discussion}

Wheat is one of the important staple crops worldwide. In China, wheat is grown on approximately 24 million hectares with a total annual production of 115 million tons and an average yield of 4.75 tons $\mathrm{ha}^{-1}[52,53]$. Wheat is very crucial for providing humans with energy. However, wheat growth is severely affected by biotic and abiotic stresses such as drought and heat. One response of the plant to drought and heat stress is to secrete wax to the epidermal layer [54]. Wax serves as a waterproof barrier and restricts the nonstomatal water loss $[18,55]$.

Biosynthesis of cuticular wax is a complex biological process that consists of two main stages: the elongation of saturated $\mathrm{C}_{16}$ and $\mathrm{C}_{18}$ fatty acyl-CoAs and the synthesis of very long-chain fatty acid, aldehydes, alcohols, alkanes, and esters. Although many wax mutants have be identified in arabidopisis [56, 57], the underlying molecular mechanism of wax biosynthesis in wheat is poorly understood. In present studies, we first determined the cuticular wax of wheat leaf blade at the seedling stage. Primary alcohols are the major components, and the $\mathrm{C}_{28}$ primary alcohol is the most abundant alcohol. These results are consistent with previously reported findings [21, 41]. Based on the micromorphology of wheat leaf blade at the seedling stage, plateletshaped wax crystals are attached to epidermal cell, and there is no difference between adaxial and abaxial sides. It is very likely that the lobed plate crystals of cuticular wax are associated with primary alcohols. Similar points were proposed by Gulz and Carver $[58,59]$.

In plants, numerous genes have been characterized that are involved in biosynthesis of wax metabolism [60, 61]. What genes are responsible for accumulation of longchain fatty alcohols in wheat? In this study, we identified and cloned three TaFARs from wheat leaf blade at the seedling stage, named TaFAR6, TaFAR7 and TaFAR8. All

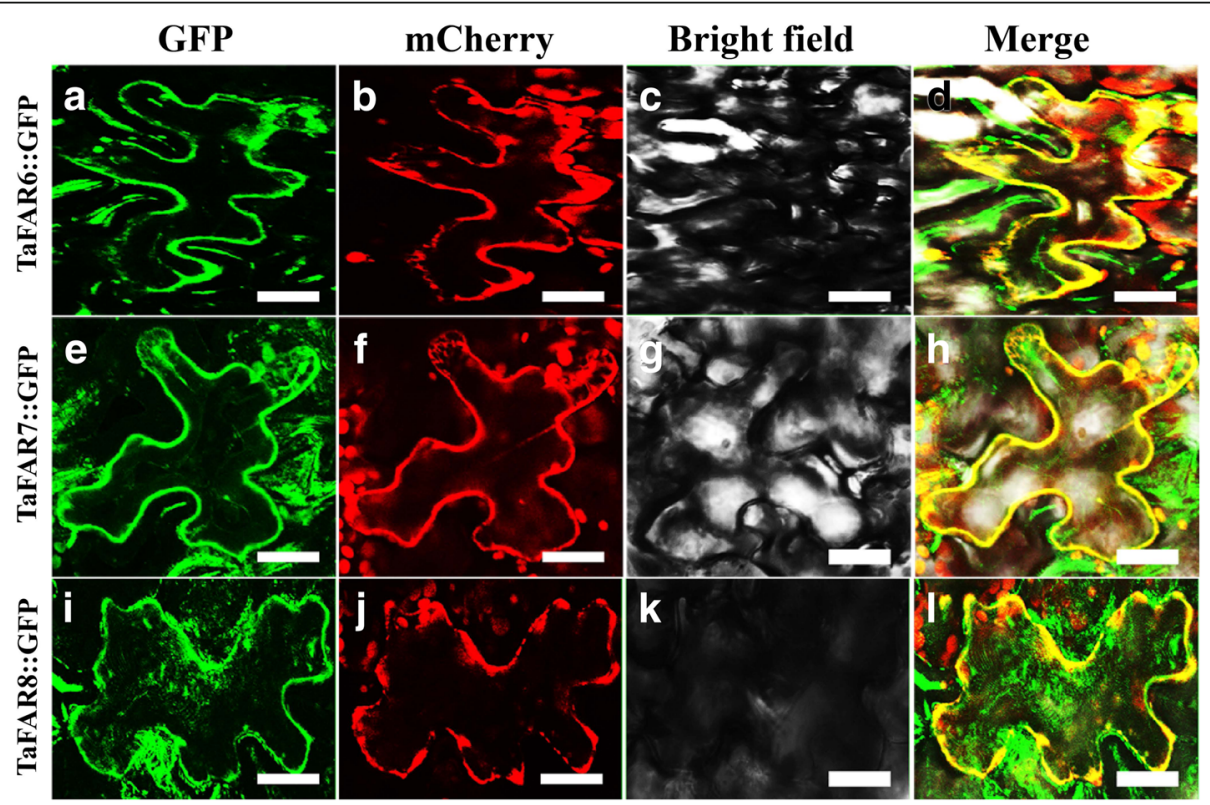

Fig. 7 Subcellular localization of TaFAR6, TaFAR7 and TaFAR8 in Nicotiana benthamiana epidermal cells. a, e, i GFP fluorescence image of the tobacco epidermal cells expressing 35S:TaFAR-GFP. b, f, j Endoplasmic reticulum (ER) marker mCherry-HDEL is indicated in red. $\mathbf{c}$, $\mathbf{g}$, $\mathbf{k}$ Bright-field image of the tobacco epidermal cells. $\mathbf{d}, \mathbf{h}$, I Merged image. Bars $=10 \mu \mathrm{m}$ 
of these genes encode a FAR with the predicted NADB domain and sterile domain. Thus, these three TaFARs likely exhibit a similar function in catalyzing primary alcohols biosynthesis. Previous studies have indicated that heterogeneous expression of CER4 in yeast results in the production of $\mathrm{C} 24: 0-\mathrm{OH}$ and $\mathrm{C} 26: 0-\mathrm{OH}$ [22]. Moreover, AtFAR1, AtFAR4 and AtFAR5 form alcohols with chain lengths ranging from C18:0 to C24:0 in yeast [26]. Expression of E.gracilis EgFAR in yeast led to the accumulation of C14:0 and C16:0 primary alcohols [42]. In hexaploid wheat, TaFAR1, TaFAR5, TaFAR2, TaFAR3 and TaFAR4 are induced to produce C22:0 or C24:0 to C28:0 primary alcohols [30, 31, 33]. To directly assess the reductase activities of the TaFARs and to investigate their substrate specificities, we expressed the coding regions of three TaFARs in yeast. All TaFAR-expressing yeast accumulated free very-long-chain fatty alcohols of the characteristic $\mathrm{C}_{24}$ and $\mathrm{C}_{26}$ length. However, the three TaFARs expressed in yeast exhibited differences. The substrate specificity of TaFAR8 was particularly stringent for $\mathrm{C}_{24}$ acyl chains. The remaining two TaFARs accept a couple of chain lengths and mostly generate $\mathrm{C} 24: 0$ and $\mathrm{C} 26: 0$ primary alcohols. These results indicated that TaFAR6, TaFAR7 and TaFAR8 have a strong preference for the C24:0 and C26:0 verylong-chain fatty acids. These TaFARs seemingly could only accept C24:0 and C26:0 fatty acyl-CoAs as substrate in yeast. This phenomenon is very similar to that of $L A G 1$, TaFAR5 and AtFAR1 [26, 31, 62].

We then performed two experiments in higher plants to further confirm the role of TaFAR6, TaFAR7 and TaFAR8 in primary alcohol biosynthesis. Transgenic expression of TaFAR6, TaFAR7 and TaFAR8 led to the accumulation of $\mathrm{C}_{24}$ to $\mathrm{C}_{32}$ primary alcohols in fruit cuticular wax of tomato and the production of $\mathrm{C}_{24}$ to $\mathrm{C}_{30}$ primary alcohols in flag leaf wax of rice. These results suggest that TaFARs may play a critical role in the biosynthesis of cuticular wax. According to the above results, it is likely that widely varying product profiles in different expression systems may be due to different cellular micro-environments between yeast and plants. Previous studies have also shown that product profiles depend largely on heterologous hosts, and thus substrate availability. For example, $\mathrm{C}_{16}$ and $\mathrm{C}_{18}$ primary alcohols were produced upon expression of jojoba FAR in E. coli, but $\mathrm{C}_{22}$ primary alcohol was detected in the seeds of high erucic acid oilseed rape (Brassica napus) plants [23]. TAA1a produced saturated $\mathrm{C}_{16}$ and monounsaturated $\mathrm{C}_{18}$ alcohols when expressed in E. coli, but saturated $\mathrm{C}_{24}$ and $\mathrm{C}_{26}$ and mono-unsaturated $\mathrm{C}_{18}$ alcohols in transgenic tobacco instead [32]. Expression of CER4 in yeast resulted in the accumulation of C24:0 and C26:0 primary alcohols, but expression of CER4 in cer4-1 produced $\mathrm{C}_{24}, \mathrm{C}_{26}, \mathrm{C}_{28}$ and $\mathrm{C}_{30}$ primary alcohols [22]. Indeed, in Arabidopsis, AtFAR3/Cer4 [22] and
AtFAR5 [26] have a strict chain length specificity and only accept a few substrates. In contrast, in wheat, six FARs (TaFAR1, TaFAR5, TAA1a, TaFAR2, TaFAR3 and TaFAR4) seemingly accept a wide range of substrates depending largely on heterologous hosts used to characterize them [30-33]. We next detect the epidermal wax by SEM. Fortunately, when TaFARs were overexpressed in higher plant, tomato and rice, waxes with increased plates were detected on the surface of fruits and leaves. These plate crystals were highly similar to the cuticular wax crystal on wheat leaf blade at the seedling stage. This finding indicates that the three TaFAR genes are expressed in transgenic lines. Previous studies demonstrated that plant cuticular wax plays various roles in harsh growth environments [63-65]. One important feature of waxes was to prevent nonstomatal water loss, which is crucial for plant survival under stress conditions. Thus, in the future, we will perform the stress experiments to confirm whether our transgenic lines could adapt to the adverse environmental abiotic factors. We also want to cultivate more drought tolerant wheat varieties via genetic engineering.

In Arabidopsis, the FAE complex generates wax synthetic precursors, and FAR enzymes produce fatty alcohols in the acyl-reduction pathway. In addition, the CER1 and MAH1 enzymes in the decarbonylation pathway [6]. Previously reported wax biosynthetic enzymes, such as OsWSL3 in rice [66], TaFAR2 in wheat [33], ZmGL8 in maize [67], BrWAX1 in cabbage [68], LeCER6 in tomato [69], as well as CsWAX1 and CsWAX2 in cucumber [70,71], are located in ER membranes. Thus, we anticipated that if these three TaFARs were involved in wax synthesis, these proteins would localize to the ER. Visualization of the functional TaFARs-GFP fusion protein in tobacco leaf epidermal cells revealed that the wheat TaFAR6, TaFAR7 and TaFAR8 are also localized to the ER. Accordingly, it is hypothesized that the ER is the subcellular compartment in which most of the cuticular wax components are deposited [57].

Numerous studies have indicated that various genes are associated with the complex regulatory network of cuticular wax biosynthesis. Most genes, Such as CER1 and CER6 in Arabidopsis [72, 73], OsGL1 in rice [74], and TaFAR5 in wheat [31], respond to water deficit, sodium chloride, cold and ABA treatments $[45,46,75]$. In our study, all six tested stimuli, including drought, heat, cold, ABA, MeJA and pathogenic fungus, induced the expression of our three TaFARs at the transcriptional level (Fig. 4), and these results were consistent with previous studies [31, 76].

In conclusion, our study presents powerful evidence that the three TaFARs encode ER-localized FARs and participate in the production of primary alcohols in cuticular wax biosynthesis of hexaploid wheat. Furthermore, 
TaFARs were associated with responses to drought, cold, heat stresses and powdery mildew infection meanwhile in MeJA and ABA-dependent manner in wheat leaves. TaFAR6, TaFAR7 and TaFAR8 are very promising genes for future genetic engineering studies aimed at generating cultivars exhibiting stress tolerance.

\section{Conclusions}

The aim of this study was to explore more functional genes involved in the biosynthesis of primary alcohols in hexaploid wheat. Overall, our results showed that TaFAR6, TaFAR7 and TaFAR8 were involved in the production of very-long-chain primary alcohols in yeast and plants, and were also associated with responses to drought, cold, heat stresses and powdery mildew infection meanwhile in MeJA and ABA-dependent manner in wheat leaves.

\section{Additional files}

Additional file 1: Table S1. Primers for vector construction and expression analysis. (XLSX $11 \mathrm{~kb}$ )

Additional file 2: Figure S2. SDS-PAGE of TaFAR6, TaFAR7 and TaFAR8 in E. coli. Arrows indicate the His-TaFAR fusion proteins. The empty vector pET28a is as control. M, protein marker. (PDF 5508 kb)

Additional file 3: Figure S3. Mass spectra of primary fatty alcohols. (a, c) The mass spectra from the products of TaFAR6, TaFAR7 and TaFAR8. (b)Authentic standard of $\mathrm{C} 24: 0-\mathrm{OH}$. (d) Authentic standard of $\mathrm{C} 26: 0-\mathrm{OH}$. (PDF $1039 \mathrm{~kb}$ )

Additional file 4: Figure S4. Genetic transformation of TaFAR6, TaFAR7 and TaFAR8 in tomato cv MicroTom and rice cv Zhonghua 11. a, Schematic representation of constructs used in the transformation experiments. LB, T-DNA left border; 355 polyA, CaMV $35 S$ polyA; Hygromycin, Hygromycin resistance gene; Tnos, NOS terminator; RB, T-DNA right border. b, Plant architecture of T1 transgenic lines at the flowering stage. C, PCR screening of transgenic T1 generation tomato plants by detecting the presence of TaFARs genes. $d$, Expression analysis of three TaFARs in different overexpression transgenic lines and CK by qRT-PCR. e, Plant architecture of T1 transgenic rice lines at the filling stage. $f, P C R$ screening of transgenic T1 generation rice plants by detecting the presence of TaFARs genes. g, Expression analysis of three TaFARs in different overexpression transgenic rice lines and CK by qRT-PCR. (PDF $10567 \mathrm{~kb}$ )

Additional file 5: Figure S5. Epicuticular wax crystal patterns on fruits and leaves of transgenic tomato detected by SEM. The epicuticular wax crystal patterns on the fruits surfaces $(a-d)$. CK (a), TaFAR6 overexpression plants (b), TaFAR7 overexpression plants (c) and TaFAR8 overexpression plants (d), respectively. The epicuticular wax crystal patterns on the leaves of adaxial surfaces (e-h) and abaxial surfaces (i-l). CK (e, i), TaFAR6 overexpression plants $(\mathrm{f}, \mathrm{j})$, TaFAR7 overexpression plants $(\mathrm{g}, \mathrm{k})$, TaFAR8 overexpression plants (h, I). CK is the empty pCXSN vector control. Scale bars $=2 \mu \mathrm{m}$. (PDF $6000 \mathrm{~kb}$ )

Additional file 6: Figure S6. Epicuticular wax crystal patterns on flag leaves and sheath of transgenic rice detected by SEM. The epicuticular wax crystal patterns on the leaves of adaxial surfaces $(a-d)$, the leaves of abaxial surfaces (e-h) and the sheath surfaces (i-l). CK plants (a, e, i), TaFAR6 overexpression plants $(b, f, j)$, TaFAR7 overexpression plants $(c, g, k)$, TaFAR8 overexpression plants $(d, h, j)$. CK is the empty pCXSN vector control. Scale bars $=2 \mu \mathrm{m}$. (PDF $9005 \mathrm{~kb}$ )

\section{Abbreviations}

ABA: Abscisic acid; FAR: Fatty acyl-coenzyme A reductase; GC-MS: Gas chromatography-mass spectrometry; kD: kilo dalton; MeJA: Jasmonic Acid Methyl ester; SEM: Scanning electron microscopy

\section{Acknowledgements}

Not applicable.

\section{Fundings}

This work was financially supported by the National Natural Science Foundation of China $(31,471,568,31,271,794)$, and Science and Technology Innovation Team Plan from shaanxi province, China (2014KCT-25). The Funding bodies were not involved in the design of the study and collection, analysis, and interpretation of data and in writing the manuscript.

\section{Availability of data and materials}

All the data pertaining to the present study has been included in table and/or figure form in the present manuscript and authors are pleased to share analyzed/raw data and plant materials upon reasonable request.

\section{Authors' contributions}

GC, ZW conceived the project and designed this research; GC, CL, FX performed the experiments with assistance from $\mathrm{YL}, \mathrm{XS}$ and $\mathrm{YW}$. All authors analyzed the data and discussed the results; GC, YW and ZW wrote the article. All the authors have read, edited, and approved the current version of the manuscript.

Ethics approval and consent to participate

Not applicable.

\section{Consent for publication}

Not applicable.

\section{Competing interests}

The authors declare that they have no competing interests.

\section{Publisher's note}

Springer Nature remains neutral with regard to jurisdictional claims in published maps and institutional affiliations.

\section{Author details}

${ }^{1}$ State Key Laboratory of Crop Stress Biology for Arid Areas, Northwest A\&F University, Yangling, Shaanxi 712100, China. ${ }^{2}$ College of Agronomy, Northwest A\&F University, Yangling, Shaanxi 712100, China.

Received: 21 September 2017 Accepted: 22 February 2018

Published online: 05 March 2018

\section{References}

1. Pandey N, labal Z, Pandey BK, Sawant SV. Phytohormones and drought stress: plant responses to transcriptional regulation. In: Pandey GK, editor. Mechanism of plant hormone signaling under stress. Hoboken: Wiley; 2017. p. 477-504.

2. Deeba F, Pandey AK, Ranjan S, Mishra A, Singh R, Sharma Y, Shirke PA, Pandey $\mathrm{V}$. Physiological and proteomic responses of cotton (Gossypium herbaceum L.) to drought stress. Plant Physiol Biochem. 2012;53:6-18.

3. Xue L, Wang P, Wang L, Renzi E, Radivojac P, Tang H, Arnold R, Zhu J-K, Tao WA. Quantitative measurement of phosphoproteome response to osmotic stress in Arabidopsis based on library-assisted extracted ion chromatogram (LAXIC). Mol Cell Proteomics. 2013;12(8):2354-69.

4. Bartels D, Sunkar R. Drought and salt tolerance in plants. Crit Rev Plant Sci. 2005;24(1):23-58.

5. Fich EA, Segerson NA, Rose JK. The plant polyester cutin: biosynthesis, structure, and biological roles. Annu Rev Plant Biol. 2016;67:207-33.

6. Kunst $L$, Samuels L. Plant cuticles shine: advances in wax biosynthesis and export. Curr Opin Plant Biol. 2009;12(6):721-7.

7. Belge B, Llovera M, Comabella E, Gatius F, Guillén P, Graell J, Lara I. Characterization of cuticle composition after cold storage of "Celeste" and "Somerset" sweet cherry fruit. J Agri Food Chem. 2014;62(34):8722-9.

8. Zhang JY, Broeckling CD, Blancaflor EB, Sledge MK, Sumner LW, Wang ZY. Overexpression of WXP1, a putative Medicago truncatula AP2 domaincontaining transcription factor gene, increases cuticular wax accumulation and enhances drought tolerance in transgenic alfalfa (Medicago sativa). Plant J. 2005:42(5):689-707.

9. Busta L, Jetter R. Structure and biosynthesis of branched wax compounds on wild type and wax biosynthesis mutants of Arabidopsis thaliana. Plant Cell Physiol. 2017;58(6):1059-74. 
10. Ahmad HM, Rahman M, Ali QAS. Plant cuticular waxes: a review on functions, composition, biosyntheses mechanism and transportation. Life Sci J. 2015;12(4s):60-7.

11. Kim KS, Park SH, Jenks MA. Changes in leaf cuticular waxes of sesame (Sesamum indicum L.) plants exposed to water deficit. J Plant Physiol. 2007;164(9):1134-43.

12. Yeats $T H$, Rose JK. The formation and function of plant cuticles. Plant Physiol. 2013;163(1):5-20.

13. Long LM, Patel HP, Cory WC, Stapleton AE. The maize epicuticular wax layer provides UV protection. Funct Plant Biol. 2003:30(1):75-81.

14. Bhushan B, Jung YC, Niemietz A, Koch K. Lotus-like biomimetic hierarchical structures developed by the self-assembly of tubular plant waxes. Langmuir. 2009;25(3):1659-66.

15. Neinhuis C, Barthlott W. Characterization and distribution of water-repellent, self-cleaning plant surfaces. Ann Bot. 1997;79(6):667-77.

16. Charnley AK. Fungal pathogens of insects: cuticle degrading enzymes and toxins. Adv Bot Res. 2003:40:241-321.

17. Haslam TM, Kunst L. Extending the story of very-long-chain fatty acid elongation. Plant Sci. 2013;210:93-107.

18. Samuels $L$, Kunst $L$, Jetter R. Sealing plant surfaces: cuticular wax formation by epidermal cells. Plant Biol. 2008;59(1):683-707.

19. Racovita RC, Jetter R. Identification of polyketides in the cuticular waxes of Triticum aestivum cv. Bethlehem Lipids. 2016;51(12):1407-20.

20. Racovita RC, Hen-Avivi S, Fernandez-Moreno J-P, Granell A, Aharoni A, Jetter R. Composition of cuticular waxes coating flag leaf blades and peduncles of Triticum aestivum cv. Bethlehem Phytochemistry. 2016;130:182-92.

21. Wang $Y$, Wang JH, Chai GQ, Li CL, Hu YG, Chen XH, Wang ZH. Developmental changes in composition and morphology of cuticular waxes on leaves and spikes of glossy and glaucous wheat (Triticum aestivum L.). PLoS One. 2015;10(10):e0141239.

22. Rowland $\mathrm{O}$, Zheng $H Q$, Hepworth SR, Lam P, Jetter R, Kunst L. CER4 encodes an alcohol-forming fatty acyl-coenzyme a reductase involved in cuticular wax production in Arabidopsis. Plant Physiol. 2006;142(3):866-77.

23. Metz JG, Pollard MR, Anderson L, Hayes TR, Lassner MW. Purification of a jojoba embryo fatty acyl-coenzyme a reductase and expression of its CDNA in high erucic acid rapeseed. Plant Physiol. 2000;122(3):635-44.

24. Aarts MG, Hodge R, Kalantidis K, Florack D, Wilson ZA, Mulligan BJ, Stiekema WJ, Scott R, Pereira A. The Arabidopsis MALE STERILITY 2 protein shares similarity with reductases in elongation/condensation complexes. Plant J. 1997;12(3):615-23.

25. Dobritsa AA, Shrestha J, Morant M, Pinot F, Matsuno M, Swanson R, Moller BL, Preuss D. CYP704B1 is a long-chain fatty acid $\omega$-hydroxylase essential for sporopollenin synthesis in pollen of Arabidopsis. Plant Physiol. 2009;151(2):574-89.

26. Domergue F, Vishwanath SJ, Joubès J, Ono J, Lee JA, Bourdon M, Alhattab R, Lowe C, Pascal S, Lessire R. Three Arabidopsis fatty acyl-coenzyme a reductases, FAR1, FAR4, and FAR5, generate primary fatty alcohols associated with suberin deposition. Plant Physiol. 2010;153(4):1539-54.

27. Shi J, Tan HX, Yu XH, Liu YY, Liang WQ, Ranathunge K, Franke RB, Schreiber L, Wang YJ, Kai GY, Shanklin J, Ma H, Zhang DB. Defective Pollen Wall is required for anther and microspore development in rice and encodes a fatty acyl carrier protein reductase. Plant Cell. 2011;23:2225-46.

28. Rowland O, Domergue F. Plant fatty acyl reductases: enzymes generating fatty alcohols for protective layers with potential for industrial applications. Plant Sci. 2012;194:28-38.

29. Wang Y, Sun YL, You QY, Luo WQ, Wang C, Zhao S, Chai GQ, Li TT, Shi X, Li $C L$, Jetter $R$, Wang $Z H$. Three fatty acyl-coenzyme a reductases, BdFAR1, BdFAR2, BdFAR3, are involved in primary alcohols biosynthesis of cuticular wax in Brachypodium Distachyon. Plant Cell Physiol. 2018; https://doi.org/10. 1093/pcp/pcx211.

30. Wang $Y$, Wang ML, Sun YL, Hegebarth D, Li TT, Jetter R, Wang ZH. Molecular characterization of TaFAR1 involved in primary alcohol biosynthesis of cuticular wax in hexaploid wheat. Plant Cell Physiol. 2015;56(10):1944-61.

31. Wang $Y$, Wang ML, Sun YL, Wang Y, Li T, Chai GQ, Jiang WH, Shan L, Li CL, Xiao ES. FAR5, a fatty acyl-coenzyme a reductase, is involved in primary alcohol biosynthesis of the leaf blade cuticular wax in wheat (Triticum aestivum L.). J Exp Bot. 2014;66:1165-75.

32. Wang A, Xia Q, Xie W, Dumonceaux T, Zou J, Datla R, Selvaraj G. Male gametophyte development in bread wheat (Triticum aestivum L.): molecular, cellular, and biochemical analyses of a sporophytic contribution to pollen wall ontogeny. Plant J. 2002:30(6):613-23.

33. Wang ML, Wang Y, Wu H, Xu J, Li TT, Hegebarth D, Jetter R, Chen LT, Wang $\mathrm{ZH}$. Three TaFAR genes function in the biosynthesis of primary alcohols and the response to abiotic stresses in Triticum aestivum. Sci Rep. 2016;6:25008.
34. Omar SC, Bentley MA, Morieri G, Preston GM, Gurr SJ. Validation of reference genes for robust qRT-PCR gene expression analysis in the rice blast fungus magnaporthe oryzae. PLoS One. 2016;11(8):e0160637.

35. Schmittgen TD, Livak KJ. Analyzing real-time PCR data by the comparative CT method. Nat Protoc. 2008;3(6):1101-8.

36. Wang ZH, Guhling O, Yao R, Li F, Yeats TH, Rose JK, Jetter R. Two oxidosqualene cyclases responsible for biosynthesis of tomato fruit cuticular triterpenoids. Plant Physiol. 2011;155(1):540-52.

37. Chetty V, Ceballos N, Garcia D, Narváez-Vásquez J, Lopez W, OrozcoCárdenas M. Evaluation of four agrobacterium tumefaciens strains for the genetic transformation of tomato(Solanum lycopersicum L.) cultivar microtom. Plant Cell Rep. 2013;32(2):239-47.

38. Hiei Y, Ohta S, Komari T, Kumashiro T. Efficient transformation of rice (Oryza sativa L.) mediated by agrobacterium and sequence analysis of the boundaries of the T-DNA. Plant J. 1994;6(2):271-82.

39. Kinkema M, Fan W, Dong X. Nuclear localization of NPR1 is required for activation of $P R$ gene expression. Plant Cell. 2000;12(12):2339-50.

40. Okuzaki A, Tabei Y. Improvement of the plastid transformation protocol by modifying tissue treatment at pre-and post-bombardment in tobacco. Plant Biotechnol J. 2012;29(3):307-10.

41. Koch K, Barthlott W, Koch S, Hommes A, Wandelt K, Mamdouh W, De-Feyter S, Broekmann P. Structural analysis of wheat wax (Triticum aestivum, CV 'Naturastar'L.): from the molecular level to three dimensional crystals. Planta. 2006;223(2):258-70.

42. Teerawanichpan P, Qiu X. Fatty acyl-CoA reductase and wax synthase from Euglena gracilis in the biosynthesis of medium-chain wax esters. Lipids. 2010;45(3):263-73.

43. Bourdenx B, Bernard A, Domergue F, Pascal S, Léger A, Roby D, Pervent M, Vile D, Haslam RP, Napier JA. Overexpression of Arabidopsis ECERIFERUM1 promotes wax very-long-chain alkane biosynthesis and influences plant response to biotic and abiotic stresses. Plant Physiol. 2011;156(1):29-45.

44. Shepherd T, Griffiths DW. The effects of stress on plant cuticular waxes. New Phytol. 2006;171(3):469-99.

45. Kosma BB, Amélie B, Eugene PP, Lü SY, Jérôme J, Matthew AJ. The impact of water deficiency on leaf cuticle lipids of Arabidopsis. Plant Physiol. 2009;151(4):1918-29.

46. Igawa T, Tokai T, Kudo T, Yamaguchi I, Kimura M. A wheat xylanase inhibitor gene, Xip-I, but not taxi-I, is significantly induced by biotic and abiotic signals that trigger plant defense. Biosci Biotechnol Biochem. 2005;69(5):1058-63.

47. Li YF, Nie YF, Zhang ZH, Ye ZJ, Zou X, Zhang L, Wang ZZ. Comparative proteomic analysis of methyl jasmonate-induced defense responses in different rice cultivars. Proteomics. 2014;14(9):1088-101.

48. Guhling $\mathrm{O}, \mathrm{Hobl} \mathrm{B}$, Yeats $\mathrm{T}$, Jetter R. Cloning and characterization of a lupeol synthase involved in the synthesis of epicuticular wax crystals on stem and hypocotyl surfaces of Ricinus communis. Arch Biochem Biophys. 2006;448(1):60-72.

49. Xia KF, Ou XJ, Gao CZ, Tang HD, Jia YX, Deng RF, Xu XL, Zhang MY. OsWS1 involved in cuticular wax biosynthesis is regulated by osa-miR1848. Plant Cell Environ. 2015;38(12):2662-73.

50. Zhang WJ, Hanisch S, Kwaaitaal M, Pedersen C, Thordal-Christensen H. A component of the Sec61 ER protein transporting pore is required for plant susceptibility to powdery mildew. Front Plant Sci. 2013;4:127.

51. Yao DS, Qi WW, Li X, Yang Q, Yan SM, Ling HL, Wang G, Wang GF, Song RT. Maize opaque10 encodes a cereal-specific protein that is essential for the proper distribution of zeins in endosperm protein bodies. PLOS Genet. 2016;12(8):e1006270

52. Liu BH, Wu L, Chen XP, Meng QF. Quantifying the potential yield and yield gap of Chinese wheat production. Agron J. 2016;108(5):1-7.

53. Chen XJ, Min DH, Yasir TA, Hu YG. Genetic diversity, population structure and linkage disequilibrium in elite Chinese winter wheat investigated with SSR markers. PLoS One. 2012;7(9):e44510.

54. Seo PJ, Lee SB, Suh MC, Park MJ, Go YS, Park CM. The MYB96 transcription factor regulates cuticular wax biosynthesis under drought conditions in Arabidopsis. Plant Cell. 2011:23(3):1138-52.

55. Jenks MA, Ashworth EN. Plant epicuticular waxes: function, production, and genetics. Hortic Rev. 1999;23:1-68.

56. Jenks MA, Rashotte AM, Tuttle HA, Feldmann KA. Mutants in Arabidopsis thaliana altered in epicuticular wax and leaf morphology. Plant Physiol. 1996;110(2):377-85.

57. Kunst $L$, Samuels A. Biosynthesis and secretion of plant cuticular wax. Prog Lipid Res. 2003;42(1):51-80. 
58. Gülz PG. Epicuticular leaf waxes in the evolution of the plant kingdom. J Plant Physiol. 1994;143(4-5):453-64.

59. Carver TLW, Curr SJ. Filamentous fungi on plant surfaces. In: Riederer M, Muller C, editors. Annual plant reviews, biology of the plant cuticle. Oxford: Blackwell; 2006. p. 368-97.

60. Lee J, Yang K, Lee M, Kim S, Kim J, Lim S, Kang G-H, Min SR, Kim S-J, Park SU. Differentiated cuticular wax content and expression patterns of cuticular wax biosynthetic genes in bloomed and bloomless broccoli (Brassica oleracea var. italica). Process Biochem. 2015;50(3):456-62.

61. Bernard A, Joubès J. Arabidopsis cuticular waxes: advances in synthesis, export and regulation. ProgLipid Res. 2013;52(1):110-29.

62. Riebeling C, Allegood JC, Wang E, Merrill AH, Futerman AH. Two mammalian longevity assurance gene (LAG1) family members, trh1 and trh4, regulate dihydroceramide synthesis using different fatty acyl-CoA donors. J Biol Chem. 2003:278(44):43452-9.

63. Lee SB, Suh MC. Advances in the understanding of cuticular waxes in Arabidopsis thaliana and crop species. Plant Cell Rep. 2015;34(4):557-72.

64. Xue DW, Zhang XQ, Lu XL, Chen GC, Chen ZH. Molecular and evolutionary mechanisms of cuticular wax for plant drought tolerance. Front Plant Sci. 2017;8:621.

65. Wang XC, Guang YY, Zhang D, Dong XB, Tian LH, Qu LQ. A $\beta$-ketoacyl-CoA synthase is involved in rice leaf cuticular wax synthesis and requires a CER2-LIKE protein as a cofactor. Plant Physiol. 2017;173(2):944-55.

66. Gan L, Wang XL, Cheng ZJ, Liu LL, Wang JL, Zhang Z, Ren YL, Lei C, Zhao ZC, Zhu SS, Wan JM. Wax crystal-sparse leaf 3 encoding a $\beta$-ketoacyl-CoA reductase is involved in cuticular wax biosynthesis in rice. Plant Cell Rep. 2016;35(8):1687-98.

67. Xu X, Dietrich CR, Lessire R, Nikolau BJ, Schnable PS. The endoplasmic reticulum-associated maize GL8 protein is a component of the acylcoenzyme a elongase involved in the production of cuticular waxes. Plant Physiol. 2002;128(3):924-34.

68. Zhang X, Liu ZY, Wang P, Wang QS, Yang S, Feng H. Fine mapping of BrWax1, a gene controlling cuticular wax biosynthesis in Chinese cabbage (Brassica rapa I.ssp.pekinensis). Mol Breeding. 2013;32(4):867-74.

69. Leide J, Hildebrandt U, Reussing K, Riederer M, Vogg G. The developmental pattern of tomato fruit wax accumulation and its impact on cuticular transpiration barrier properties: effects of a deficiency in a $\beta$-ketoacylcoenzyme a synthase (LeCER6). Plant Physiol. 2007;144(3):1667-79.

70. Wang WJ, Zhang Y, Xu C, Ren JJ, Liu XF, Black K, Gai XS, Wang Q, Ren HZ Cucumber ECERIFERUM1 (CSCER1), which influences the cuticle properties and drought tolerance of cucumber, plays a key role in VLC alkanes biosynthesis. Plant Mol Biol. 2015;87(3):219-33.

71. Wang WJ, Liu XF, Gai XS, Ren JJ, Liu X, Cai Y, Wang Q, Ren HZ. Cucumis sativus L. WAX2 plays a pivotal role in WAX biosynthesis, influencing pollen fertility and plant biotic and abiotic stress responses. Plant Cell Physiol. 2015;56(7):1339-54.

72. Aarts M, Keijzer CJ, Stiekema WJ, Pereira A. Molecular characterization of the CER1 gene of Arabidopsis involved in epicuticular wax biosynthesis and pollen fertility. Plant Cell. 1995;7(12):2115-27.

73. Hooker TS, Millar AA, Kunst L. Significance of the expression of the CER6 condensing enzyme for cuticular wax production in Arabidopsis. Plant Physiol. 2002;129(4):1568-80.

74. Islam MA, Du H, Ning J, Ye H, Xiong L. Characterization of Glossy 1homologous genes in rice involved in leaf wax accumulation and drought resistance. Plant Mol Biol. 2009;70(4):443-56.

75. Wang YH, Wan LY, Zhang LX, Zhang ZJ, Zhang HW, Quan RD, Zhou SR, Huang RF. An ethylene response factor OsWR1 responsive to drought stress transcriptionally activates wax synthesis related genes and increases wax production in rice. Plant Mol Biol. 2012;78(3):275-88.

76. Lin RM, Zhao WS, Meng XB, Peng YL. Molecular cloning and characterization of a rice gene encoding AP2/EREBP-type transcription factor and its expression in response to infection with blast fungus and abiotic stresses. Physiol Mol Plant Pathol. 2007;70(1):60-8. 